\title{
Coordinate Transformation is First Completed Downstream of Primary Motor Cortex
}

\author{
Yuval Yanai, ${ }^{1}$ Nofya Adamit, ${ }^{1}$ Zvi Israel, ${ }^{2}$ Ran Harel, ${ }^{3}$ and Yifat Prut ${ }^{1}$ \\ ${ }^{1}$ Department of Physiology and the Interdisciplinary Center for Neural Computation, Hadassah Medical School, The Hebrew University, Jerusalem 91120 \\ Israel, ${ }^{2}$ Department of Neurosurgery, Hadassah Hospital, Jerusalem 91120, Israel, and ${ }^{3}$ Department of Neurosurgery, Sheba Medical Center, Tel Aviv 52621, \\ Israel
}

\begin{abstract}
It was suggested previously that the transformation of action to muscle-based coding is completed in the primary motor cortex (M1). This is consistent with a predominant direct pathway leading from M1 to motoneurons. Accordingly, spinal segmental interneurons that are located downstream to M1 are expected to show muscle-like coding properties. We addressed this hypothesis using simultaneous recording of cortical and spinal activity in primates performing an isometric wrist task with multiple targets and two hand postures. Here we show that while the motor cortex follows an intermediate coordinate frame, spinal interneurons already follow a muscle-like coordinate frame. We thus suggest that the final steps in coordinate transformation of motor commands take place downstream of M1 via corticospinal interactions.
\end{abstract}

Key words: motor cortex; spinal cord; directional tuning; coordinate frames; EMG; motor control

\section{Introduction}

Moving and interacting with the environment require an ongoing translation of information obtained in sensor-defined coordinates into commands defined in effector coordinates (Pouget et al., 2000). Single-unit and population activity in sensory and motor cortices were found to be related to vector properties (direction and amplitude) of a stimulus or an action (Georgopoulos et al., 1982; Andersen et al., 1985). These findings led researchers to associate specific coordinate frames with different cortical areas. A previous study (Kakei et al., 1999, 2001) used a task manipulation that dissociated coordinate frames to show that premotor ventral neuronal activity was consistently related to an extrinsic (target-like) coordinate frame, whereas neuronal activity in M1 consisted of two distinct subpopulations: extrinsic and muscle-like neurons. The coexistence of these two systems in a single site prompted the hypothesis that $\mathrm{M} 1$ is responsible for the translation of coordinate frames from extrinsic to muscle-like. This suggestion is in accordance with the direct influence of motor cortex on spinal motoneurons (Todorov, 2000; Lemon et al., 2004), although it has been argued that even this direct corticomotoneuronal path is subjected to further processing at the spinal level (McKiernan et al., 2000; Schieber and Rivlis, 2007). The natural prediction is that spinal interneurons (INs), which are located downstream of the motor cortex, operate in a muscle-like coordinate frame. Using a similar task we confirm this prediction, but show that the hypothesis may require some refinements.

Received 0ct. 14, 2007; revised Nov. 25, 2007; accepted Dec. 18, 2007.

This work was supported by Israel Science Foundation Grants ISF-555/01 and ISF-1355/05 and by the Baruch Foundation. We thank Steve Perlmutter for useful comments and help.

Correspondence should be addressed to Yifat Prut, Department of Physiology, Hadassah Medical School, The Hebrew University, P.0. Box 12272, Jerusalem 91120, Israel. E-mail: yifatpr@ekmd.huji.ac.il.

DOI:10.1523/JNEUROSCI.4662-07.2008

Copyright $\odot 2008$ Society for Neuroscience $\quad$ 0270-6474/08/281728-05\$15.00/0
Here, we show that although INs appeared closely related to a muscle-based representation as initially predicted, the motor cortex operates in a coordinate frame that is neither extrinsic nor intrinsic. These results indicate that corticospinal interactions participate in the emergence of muscle-based representations of motor commands. Spinal INs are thus an ideal candidate to serve as a processing station for the cortical command before it reaches muscles (Alstermark and Isa, 2002).

\section{Materials and Methods}

Animals and behavioral task. Three monkeys (Maccaca fascicularis) performed an isometric wrist task with an instructed delay period. The monkeys held their hand in either a pronation or supination position and controlled a cursor on a computer screen by applying a two-dimensional (2D) isometric torque at the wrist. A trial was initiated by the appearance of a central target. The monkey positioned the cursor inside the target by generating zero torque for a rest period $(0.5 \mathrm{~s})$. Then, eight peripheral targets appeared (uniformly distributed around the center target at a fixed distance) with one target changing its color for $0.5 \mathrm{~s}$ (cue), defining the onset of a delay period. The disappearance of the central target (0.8$1.7 \mathrm{~s}$ after cue onset) served as a "go" signal. The monkeys then had to attain the previously filled target by generating an isometric twodimensional torque in the appropriate direction, and keep the cursor within the target box for an active-hold period $(0.35-0.7 \mathrm{~s})$. Subsequently, the peripheral targets disappeared and the central target reappeared. The monkey returned to the rest position and received a reward, after which the screen went blank for 1-1.5 s and a new trial started.

Recording sessions. Details of the recording technique are described previously (Prut and Perlmutter, 2003). A cortical chamber was implanted above the motor cortex and the location of arm-related primary motor cortex was mapped using a train of stimulating pulses $(50 \mathrm{~ms}$ of biphasic stimulation given at $300 \mathrm{~Hz}$ with intensity $\leq 60 \mu \mathrm{A}$ ). Subsequently, a spinal chamber was implanted above the cervical spinal cord (C6-T1). Extracellular single-unit activity was recorded from motor cortex and spinal interneurons located at intermediate lamina while the 
monkey performed the task. After recordings were completed, two of the monkeys were deeply anesthetized (ketamine, pentobarbital, $30 \mathrm{mg} / \mathrm{kg}$ ) and pins were inserted into known coordinates of the cortical implant. Animals were then killed with pentobarbital sodium $(50 \mathrm{mg} / \mathrm{kg}$, i.v.) and perfused with $4 \%$ paraformaldehyde. Cortical locations of penetrations relative to anatomical landmarks were subsequently reconstructed. All surgical and animal handling procedures were according to the National Institutes of Health Guide for the Care and Use of Laboratory Animals (1996), complied with Israeli law, and were approved by the Ethics Committee of the Hebrew University. Animal care was supervised by the veterinarian staff of the Hebrew University.

Data collection. We recorded single-unit activity from arm- and handrelated areas in the motor cortex of three monkeys. We found 207 neurons that were directionally sensitive in at least one hand posture during torque ramp (defined as $0 \mathrm{~ms}$ before until $500 \mathrm{~ms}$ after torque onset time). Two other epochs were defined as "hold," defined from $500 \mathrm{~ms}$ before until the beginning of return to center position, and "pretorque" (PT) defined from $500 \mathrm{~ms}$ before until torque onset time. Of this population, 135 neurons were directionally tuned in both hand postures during torque ramp. Of 135 cortical neurons that were tuned in both hand postures during torque ramp, 82 were recorded from sites in which intracortical microstimulation evoked hand/wrist movements and were thus labeled as "hand related neurons." In two monkeys, we recorded single-unit activity from segmental (C6-T1) spinal interneurons. We found 77 neurons that were directionally sensitive in at least one hand posture during torque ramp and 36 spinal neurons that were directionally tuned in both hand postures. We recorded electromyogram activity from selected identified forearm muscles (number of samples in parentheses): flexor carpi radialis (2), flexor carpi ulnaris (1), flexor diagitorum superficialis (3), flexor digitorum profundus (3), palmaris longus (3), extensor carpi radialis (2), extensor carpi ulnaris (1), extensor digitorum communis (2), extensor digitorum 2, 3 (1), and extensor digitorum 4,5 (3). All muscles were directionally tuned in both hand postures during torque ramp.

Data analysis. For each time window, we analyzed neurons that were directionally tuned in both hand postures. Directional tuning was determined using a bootstrap method (Crammond and Kalaska, 2000) $(p<$ 0.05). Two preferred direction (PD) shift distributions were compared by a test for common mean direction of two samples (Fisher, 1993).

A dynamic index (DI) was computed to quantify posture-related changes in the level of force-direction firing of single-cells (Sergio and Kalaska, 2003): $\mathrm{DI}=(D R \mathrm{p}-D R \mathrm{~s}) /(D R \mathrm{p}+D R \mathrm{~s}) \cdot D R \mathrm{p}$ and $D R \mathrm{~s}$ were defined as the difference between the maximal versus minimal targetrelated firing in pronation and supination, respectively. Thus, DI ranged from -1 to +1 . Large absolute values indicate large posture-dependent differences in firing rate. Near-zero values indicate no change in the dynamic range of firing induced by a change in wrist configuration.

The population vector reconstruction was based on vector summation (Sergio et al., 2005):

$$
P(M, p)=\sum_{i} w_{i}(M, p) C_{i}(r)
$$

where $P$ is a vector pointing in the reconstructed direction for target $M$ $(M=[1 \ldots 8])$ and hand posture $p$ (pronation or supination), $C i(r)$ is the preferred direction of neuron $i$ at wrist posture $r$ (pronation or supination) during torque ramp, and $w i(M, p)$ is a weight computed for each target $M$ and hand posture $p$ using $w_{i}(M, p)=d_{i}(M, p)-\bar{d}_{i, \text { rest }}(p)$. $d_{i}(M, p)$ is the average firing rate of neuron $i$ in the $M$ direction at the $p$ hand posture during torque ramp. $\bar{d}_{i, \text { rest }}(p)$ is the average firing rate of neuron $i$ at the $p$ hand posture across all targets during the rest period (500 ms before cue onset). If $r=p$, then the reconstruction uses weights and PDs from the same hand posture, whereas if $r \neq p$, then the reconstruction uses weights from the current hand posture with PDs of the opposite hand posture.

\section{Results}

We trained three monkeys to perform a 2D isometric center-out wrist task to eight directions in two hand postures: pronation and
Table 1. Mean PD shifts expressed by neurons and muscles at different epochs

\begin{tabular}{llll}
\hline & Pretorque $(n$, mean $\Delta P D)$ & Torque ramp & Hold \\
\hline Muscles & $10,-64^{\circ}$ & $21,-75^{\circ}$ & $21,-75^{\circ}$ \\
Spinal & $18,-50^{\circ} a$ & $36,-65^{\circ}$ & $36,-63^{\circ}$ \\
Cortex & $93,-27^{\circ}$ & $135,-43^{\circ}$ & $85,-52^{\circ}$ \\
Cortex: hand & $55,-33^{\circ}$ & $82,-54^{\circ}$ & $48,-53^{\circ}$ \\
\hline
\end{tabular}

${ }^{a}$ During pretorque, the distribution of spinal PD shifts appeared bimodal; hence, the mean is less informative.

supination. Consistent with a previous study, we found that this task dissociates three coordinate frames locked to either: extrinsic $\left(0^{\circ}\right.$ rotation $)$, muscle activity $\left(\sim 75^{\circ}\right.$ clockwise rotation $)$, or handorientation $\left(180^{\circ}\right.$ rotation; previously referred to as the joint coordinate system). We recorded single-unit activity from 207 taskrelated cells in arm-related areas of the motor cortex in three monkeys and from 77 task-related segmental interneurons in the cervical enlargement of two monkeys. Of these cells and for any given time window we took the subgroup of cells that were directionally tuned in both hand postures and used the shift in their preferred directions $(\Delta \mathrm{PD})$ between hand postures to assign an appropriate coordinate frame.

The magnitude and distribution of PD shifts computed for both spinal and cortical neurons, but not forearm muscles, varied across the different epochs included in the analysis (Table 1). Before torque onset (PT period), the distribution of $\Delta \mathrm{PDs}$ of cortical neurons was predominantly unimodal with a consistently (i.e., nonrandom) clockwise PD shift that was in between extrinsic (i.e., no shift in PD) and muscles. This distribution was significantly different from the $\Delta \mathrm{PD}$ distribution of the forearm muscles $[p<0.05$, test for common mean direction for two samples (CMD2S)]. These results were also confirmed for a subgroup of cortical neurons that were recorded from hand-related sites (supplemental Fig. 1, available at www.jneurosci.org as supplemental material). At the same time, tuned spinal neurons exhibited a bimodal distribution of PD shifts with one subgroup showing a clockwise PD shift similar to the mean cortical shift $(n=7$, $-6^{\circ}<\Delta \mathrm{PD}<-32^{\circ}$; mean, $-18^{\circ}$ ) and a second subgroup showing a larger $\mathrm{PD}$ shift $\left(n=9,-55^{\circ}<\Delta \mathrm{PD}<-115^{\circ}\right.$; mean, $\left.-75^{\circ}\right)$ comparable with the shift observed for forearm muscles.

When monkeys started to exert torque (torque ramp), a larger number of cortical and spinal neurons were tuned in both hand configurations, and the observed $\Delta \mathrm{PD}$ s were larger. At this time, the spinal $\triangle \mathrm{PD}$ distribution was unimodal and was not significantly different from the $\Delta \mathrm{PD}$ distribution of the forearm muscles $(p>0.5, \mathrm{CMD} 2 \mathrm{~S})$. The cortical $\Delta \mathrm{PD}$ s also increased, but remained significantly different from both spinal neurons $(p<$ $0.05, \mathrm{CMD} 2 \mathrm{~S})$ and forearm muscles $(p<0.05, \mathrm{CMD} 2 \mathrm{~S})$. Note that the distribution of cortical PD shifts was broad and slightly bimodal. Nevertheless, most of these neurons followed an intermediate coordinate frame $\left(n=76,56 \%,-60^{\circ}<\Delta \mathrm{PD}<15^{\circ}\right.$; mean, $-28^{\circ}$ ). Only $23 \%$ of the cells had a PD shift comparable with a muscle coordinate frame $\left(n=31,-135^{\circ}<\Delta \mathrm{PD}<-60^{\circ}\right.$; mean, $\left.-95^{\circ}\right)$. Hand-related cortical neurons were not significantly different from spinal neurons ( $p>0.3$, CMD2S) or forearm muscles ( $p>0.08, \mathrm{CMD} 2 \mathrm{~S})$. However, for this same group of neurons, a larger number of cells followed an intermediate coordinate frame $\left(n=40 / 82 ;\right.$ mean, $\left.30^{\circ}\right)$ compared with a muscle coordinate frame $\left(n=25 / 82\right.$; mean, $\left.-92^{\circ}\right)$.

During the last $500 \mathrm{~ms}$ of the hold period, the distributions of PD shifts were broader. The number of participating muscle and spinal INs remained the same as during torque ramp, but the number of available cortical neurons decreased substantially. Ap- 
parently, at this period the two coordinate systems (cortical and spinal) tended to coalesce while the monkey was required to exert a steady directional torque. No significant differences were found between spinal cord and the cortex $(p>0.2, \mathrm{CMD} 2 \mathrm{~S})$ and between spinal cord and muscles $(p>$ 0.2 , CMD2S). However, the difference between cortex and muscles remained significant $(p<0.05, \mathrm{CMD} 2 \mathrm{~S})$.

The process of transition of extrinsicto-intrinsic representation along task performance was further examined using a sliding window of $300 \mathrm{~ms}$ that was moved in $100 \mathrm{~ms}$ steps (Fig. 1D). After cue onset, only a few spinal and cortical cells were tuned and both populations expressed a near extrinsic-based coding (mean $\triangle \mathrm{PD}$, $\sim 20^{\circ}$ clockwise). Soon after, spinal representation became muscle-like, whereas cortical neurons maintained an intermediate representation. The two systems only converged during the late phases of the performed action where they expressed a near muscle-based representation. Interestingly, this convergence appeared transient, after which cortical neurons returned to exhibit an intermediate representation, whereas spinal neurons (and muscles) maintained a complete PD shift.

The discrepancy in PD shifts between motor cortex and muscles is not necessarily at odds with direct cortical control of movement, because of the posturedependent modulation in firing rates (Andersen et al., 1985; Kakei et al., 2003) (see example of polar plots computed for spinal neuron in Fig. $1 B$ ). It was shown theoretically that premotoneuronal neurons can directly control muscles using a strategy that exchanges PD shifts for a dynamic range of firing (Salinas and Abbott, 1995; Shah et al., 2004). Nevertheless, we found that during torque ramp the dynamic indices (see Materials and Methods) of cortical and spinal neurons were both symmetrically distributed around zero (Fig. 1E). Thus, despite having different PD shifts, both cortical and spinal neurons expressed a similar modulation of their dynamic ranges.

Although intact spinal machinery properly decodes the intermediate cortical coordinate frame, it may pose a problem for artificial decoding algorithms (e.g., the population vector) (Georgopoulos et al., 1986) if it is assumed that the population signal relates to a fixed coordinate frame (extrinsic or intrinsic). We found that using the population vector (Sergio et al., 2005) with the appropriate PDs resulted in very accurate direction estimations as long as we kept PD estimation and movement reconstruction within a single hand posture (Fig. 2). However, reconstructing direction after changing the hand posture without updating the PD caused an obvious error in
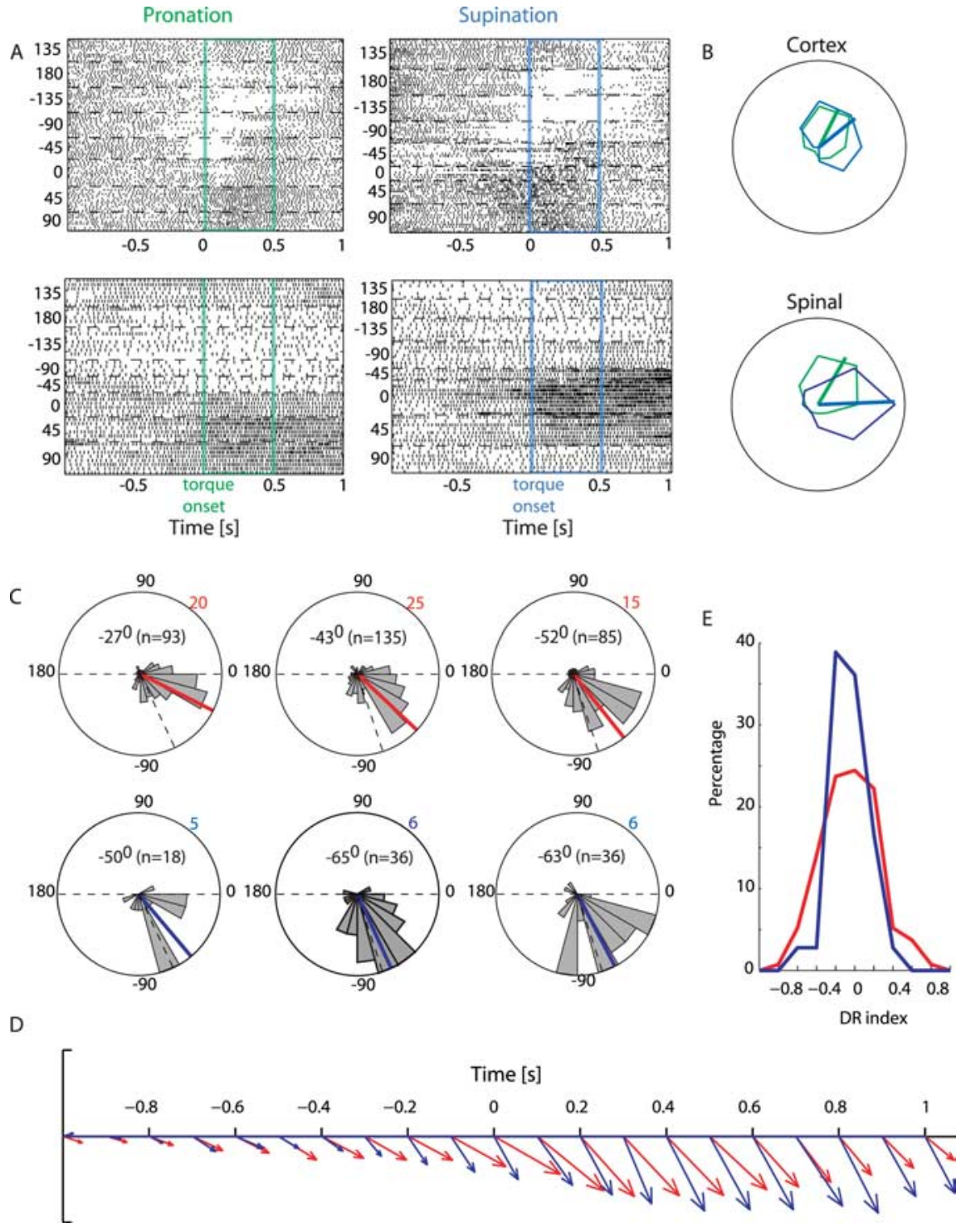

Figure 1. Consistent albeit different PD shifts in the motor cortex and in the spinal cord. $A$, Raster plots of a cortical neuron (up) and a spinal interneuron (down) aligned on torque onset in two hand postures: pronation (left) and supination (right). $\boldsymbol{B}$, Polar plots of directional tuning and preferred directions during torque ramp for the same cortical neuron (up) and spinal interneuron (down) computed for pronation (green) and supination (blue) trials. The clockwise shift of PD with hand posture can be observed. In the examined time window the spinal interneuron showed a gain field effect. $\boldsymbol{C}$, Polar histograms of the PD shifts of motor cortex neurons (up) and INs (down) during the 500 ms before torque onset (left), after torque onset (middle) and before release of active torque (right). Dashed lines mark the extrinsic $\left(0^{\circ}\right)$, hand-orientation $\left(180^{\circ}\right)$ and muscle (mean $\left.\Delta P D\right)$ coordinate frames. The shifts are consistently clockwise with a clear difference in values between motor cortex and downstream structures around torque onset. The PD shifts in the motor cortex and spinal cord become more similar but more variable during active hold. $D$, Moment-to-moment mean $\triangle P D$ in the cortex (red) and spinal cord (blue). Both structures are similar during early delay and late ramp periods. However, the two structures depart in their representation during late delay and early ramp periods. The length of the arrows is proportional to the number of units participating (normalized by the maximum number of participating units in each structure). The number of participating units in the motor cortex decreases during late ramp period but remains steady in the spinal cord. $\boldsymbol{E}$, Dynamic indices in the motor cortex (red) and spinal cord (blue) are symmetrically distributed around zero.

direction estimation because of the consistent PD shift exhibited by cortical and spinal neurons.

\section{Discussion}

Our results demonstrate that around torque-onset, motor cortex and structures located downstream to it do not share a common coordinate frame. Spinal INs appeared to follow a muscle-based coordinate frame early in the task, whereas the emergence of a small proportion of muscle-like cortical neurons appeared later 


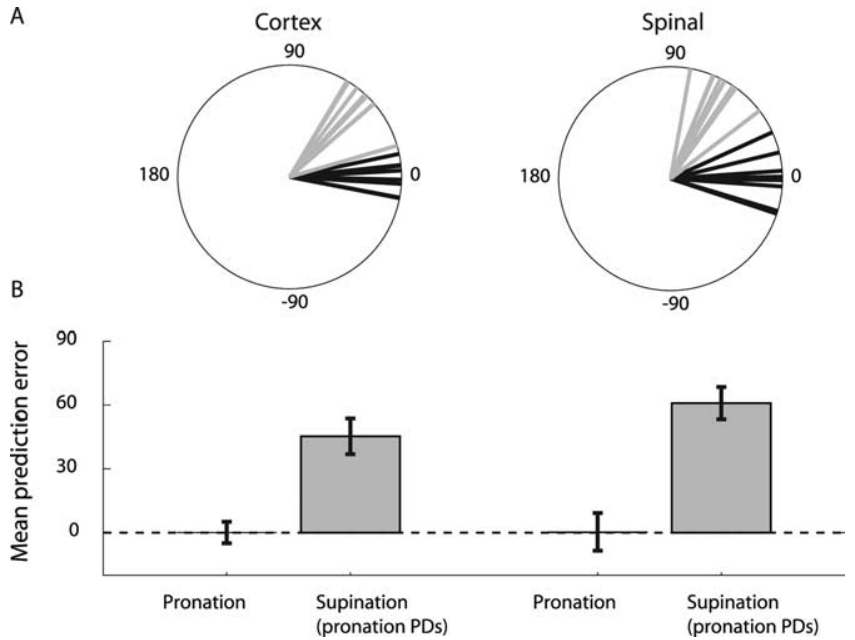

Figure 2. Change of posture produces errors in directional reconstruction. $A$, Polar plots of directional error to eight targets during ramp using the population vector method in the motor cortex (left) and in the spinal cord (right). Predicting direction using pronation PDs was accurate when the hand was indeed in pronation posture (black). However, there was a systematic bias in directional prediction when using pronation PDs while the hand was in supination (gray). $\boldsymbol{B}$, Summary of the mean and circular STD of the directional errors during torque ramp. The bias in the prediction in both the cortex and spinal cord was of similar magnitude and opposite sign to the mean PD shift in each structure.

in the task during motor execution. The majority of cortical neurons, however, appeared to follow a coordinate frame that was somewhere in between the extrinsic and the intrinsic muscle-like coordinate frames throughout the task. This suggests that cortical commands are further processed downstream, possibly via a segmental network dynamic (Deneve et al., 2001) and/or a specific wiring scheme of descending pathways on motoneurons.

Before torque onset, cortical neurons showed a consistent but partial PD shift which differed from the bimodal distribution of PD shifts (no shift and complete shift) observed in the reference study (Kakei et al., 1999). A possible source for this discrepancy is the isometric task used in our study (Sergio et al., 2005). In fact, we observed slower cursor motion (mean, $670 \mathrm{~ms}$ ), a monotonic increase in muscle activation, and a steady torque rather than a steady position at the peripheral target. In contrast, the movement task was very fast (movement time, $<200 \mathrm{~ms}$ ) and had a more complex muscle activation pattern. It might therefore engage a pure feedforward control in which M1 exerts predominant direct control.

Posture-dependent PD shifts were observed previously in motor cortex in tasks involving the whole arm (Scott and Kalaska, 1997). The shifts appeared consistent when the arm was rotated around an axis that was perpendicular to the work plane (Caminiti et al., 1991; Sergio and Kalaska, 2003; Morrow et al., 2007). However, the use of a larger rotation of the hand $\left(180^{\circ}\right)$ enabled dissociation between the external, muscle, and hand-orientation representations that was difficult to obtain in previous studies. Furthermore, it allowed us to observe the differential effect on cortical and spinal populations. Because both cortical and spinal neurons showed a similar modulation of dynamic range, we suggest that the magnitude of observed PD shifts is not a redundant feature, but a fundamental functional property of the neural computations taking place in each of these premotoneuronal circuitries. Partial PD shifts were suggested to play a role in coordinate transformation by stochastic recurrent neural networks (Deneve et al., 2001). Our results tend to support such a cortical mechanism for control of movements. In addition, our results suggest that as trials progress, cortical command changes as a result of different weighing of extrinsic-related with musclerelated inputs. Spinal circuitry may thus be required to transform this variable cortical command into a consistent muscle command.

Previous studies have shown that using the activity of a population of neurons to reconstruct movement has the advantage of reducing the variability associated with the firing rate of a single neuron (Schwartz et al., 2006). However, as expected and as shown by our results, a consistent bias cannot be washed out by the population averaging. For example, in our work using a model that was constructed for one hand posture to predict direction in a different hand posture led to a prediction that was incompatible with extrinsic, hand-orientation, or muscle-related directions. It thus appears that reconstruction success may be confined to a specific motor context and attempts to extrapolate these results to a different motor context may yield poor performance. Because the term motor context may include internal or mental processes (Georgopoulos et al., 1989), which can be harder to control, a correct reconstruction of motor action based on cortical signals may require adaptive control algorithms (Taylor et al., 2002).

\section{References}

Alstermark B, Isa T (2002) Premotoneuronal and direct corticomotoneuronal control in the cat and macaque monkey. Adv Exp Med Biol 508:281-297.

Andersen RA, Essick GK, Siegel RM (1985) Encoding of spatial location by posterior parietal neurons. Science 230:456-458.

Caminiti R, Johnson PB, Galli C, Ferraina S, Burnod Y (1991) Making arm movements within different parts of space: the premotor and motor cortical representation of a coordinate system for reaching to visual targets. J Neurosci 11:1182-1197.

Crammond DJ, Kalaska JF (2000) Prior information in motor and premotor cortex: activity during the delay period and effect on pre-movement activity. J Neurophysiol 84:986-1005.

Deneve S, Latham PE, Pouget A (2001) Efficient computation and cue integration with noisy population codes. Nat Neurosci 4:826-831.

Fisher NI (1993) Statistical analysis of circular data. Cambridge, UK: Cambridge UP.

Georgopoulos AP, Kalaska JF, Caminiti R, Massey JT (1982) On the relations between the direction of two-dimensional arm movements and cell discharge in primate motor cortex. J Neurosci 2:1527-1537.

Georgopoulos AP, Schwartz AB, Kettner RE (1986) Neuronal population coding of movement direction. Science 233:1416-1419.

Georgopoulos AP, Lurito JT, Petrides M, Schwartz AB, Massey JT (1989) Mental rotation of the neuronal population vector. Science 243:234-236.

Kakei S, Hoffman DS, Strick PL (1999) Muscle and movement representations in the primary motor cortex. Science 285:2136-2139.

Kakei S, Hoffman DS, Strick PL (2001) Direction of action is represented in the ventral premotor cortex. Nat Neurosci 4:1020-1025.

Kakei S, Hoffman DS, Strick PL (2003) Sensorimotor transformations in cortical motor areas. Neurosci Res 46:1-10.

Lemon RN, Kirkwood PA, Maier MA, Nakajima K, Nathan P (2004) Direct and indirect pathways for corticospinal control of upper limb motoneurons in the primate. Prog Brain Res 143:263-279.

McKiernan BJ, Marcario JK, Karrer JH, Cheney PD (2000) Correlations between corticomotoneuronal (CM) cell postspike effects and cell-target muscle covariation. J Neurophysiol 83:99-115.

Morrow MM, Jordan LR, Miller LE (2007) Direct comparison of the taskdependent discharge of M1 in hand space and muscle space. J Neurophysiol 97:1786-1798.

Pouget A, Dayan P, Zemel R (2000) Information processing with population codes. Nat Rev Neurosci 1:125-132.

Prut Y, Perlmutter SI (2003) Firing properties of spinal interneurons during voluntary movement. I. State-dependent regularity of firing. J Neurosci 23:9600-9610. 
Salinas E, Abbott LF (1995) Transfer of coded information from sensory to motor networks. J Neurosci 15:6461-6474.

Schieber MH, Rivlis G (2007) Partial reconstruction of muscle activity from a pruned network of diverse motor cortex neurons. J Neurophysiol 97:70-82.

Schwartz AB, Cui XT, Weber DJ, Moran DW (2006) Brain-controlled interfaces: movement restoration with neural prosthetics. Neuron 52:205-220.

Scott SH, Kalaska JF (1997) Reaching movements with similar hand paths but different arm orientations. I. Activity of individual cells in motor cortex. J Neurophysiol 77:826-852.

Sergio LE, Kalaska JF (2003) Systematic changes in motor cortex cell activity with arm posture during directional isometric force generation. J Neurophysiol 89:212-228.

Sergio LE, Hamel-Paquet C, Kalaska JF (2005) Motor cortex neural correlates of output kinematics and kinetics during isometric-force and armreaching tasks. J Neurophysiol 94:2353-2378.

Shah A, Fagg AH, Barto AG (2004) Cortical involvement in the recruitment of wrist muscles. J Neurophysiol 91:2445-2456.

Taylor DM, Tillery SI, Schwartz AB (2002) Direct cortical control of 3D neuroprosthetic devices. Science 296:1829-1832.

Todorov E (2000) Direct cortical control of muscle activation in voluntary arm movements: a model. Nat Neurosci 3:391-398. 